\title{
Hunting for a pleural fluid test for mesothelioma: is soluble mesothelin the answer?
}

\section{Y C Gary Lee}

A n estimated 150000 patients develop a pleural effusion each year in the UK. Establishing the aetiology of pleural effusions can be challenging as they can be associated with over 50 systemic or pulmonary disorders. ${ }^{1}$ Exudative pleural fluids arise from plasma extravasation and contain proteins and cells from the systemic circulation, as well as those released locally from the pleura. This milieu of cellular products potentially holds vital clues that can unveil the underlying cause of the effusion. Decoding these clues has been a long and slow journey, ${ }^{2}$ and only in recent years have several disease-specific pleural fluid markers been incorporated into clinical practice (eg, adenosine deaminase for tuberculous pleuritis ${ }^{3}$ and brain natriuretic peptide for effusions from cardiac failure).

It is logical that diagnostic clues for mesothelioma, a primary pleural malignancy, would rest within the pleural fluid. The incidence of mesothelioma is rising significantly in the UK and other European countries. Most patients with mesothelioma present with a pleural effusion, and this diagnosis should be considered in all patients with exudative effusions. However, fluid cytology has a notoriously low diagnostic yield, as differentiating mesothelioma cells from benign (or reactive) mesothelial cells is difficult. More invasive procedures such as thoracoscopy for tissue biopsy samples are frequently required. ${ }^{4}$

Finding a diagnostic marker for mesothelioma is a challenging endeavour. ${ }^{5}$ This is made difficult by the heterogeneity of mesothelioma, which comprises various histological subtypes (eg, epithelioid, sarcomatoid, desmoplastic) with dissimilar gene expression patterns, ${ }^{6}$ phenotypes and biological features. ${ }^{4}$ No unique molecule has been shown to reliably define mesothelioma from benign mesothelium or metastatic carcinomas, even by profiling the expression of tens of thousands of genes on mesothelioma tissues using microarray technology.

\section{SOLUBLE MESOTHELIN IN MESOTHELIOMA}

The Food and Drug Administration has recently approved the use of soluble mesothelin for disease monitoring in mesothelioma. This followed the findings of Robinson et al that blood levels of soluble mesothelin are significantly raised in patients with mesothelioma compared with controls with a wide range of pleuropulmonary diseases. This observation is robust and has been confirmed by

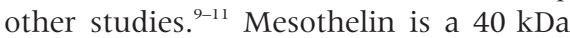
glycoprotein predominantly found in normal mesothelial cells in the pleura, peritoneum and pericardium. Although mesothelin is bound to cell membrane, a circulating form termed soluble mesothelin-related peptides (SMRP or "soluble mesothelin") has been found in the serum. It has been suggested that the protein may be a splice variant of the normal mesothelin, with structural variation that alters its binding to cells. ${ }^{12}$ More recently it has been suggested that SMRP are mesothelin molecules released into the systemic circulation from malignant cells that overexpress mesothelin. ${ }^{13}{ }^{14}$

Most (>95\%) patients with mesothelioma have a pleural effusion; it would be logical to assess the usefulness of soluble mesothelin in pleural fluid as a diagnostic test for mesothelioma. In this issue of Thorax, Creaney et $a l^{15}$ report that soluble mesothelin is detectable in pleural and peritoneal effusions and its levels are significantly higher (median 7-fold) in pleural mesothelioma $(n=52)$ than in effusions of benign pleuritis $(n=84)$ and non-mesothelioma malignancies $(\mathrm{n}=56)$ (see page 569). Levels of soluble mesothelin were also higher in ascitic fluid from peritoneal mesothelioma than in benign controls.

Using a cut-off that yielded $98 \%$ specificity, the sensitivity of raised soluble mesothelin in pleural fluid was $67 \%$ for all pleural mesothelioma and $77 \%$ for epithelioid mesothelioma. The results from the study by Creaney et al ${ }^{15}$ echoed those from a multicentre study from France which compared mesothelin levels in pleural fluid from patients with mesothelioma, metastatic carcinomas and benign asbestos pleural effusions. Scherpereel et al ${ }^{9}$ found a similar sensitivity $(65 \%)$ for pleural fluid mesothelin with a specificity of $84 \%$. To put this in context, soluble mesothelin has a much more favourable sensitivity than other tumour markers adopted in routine clinical practice, including prostate specific antigen $(21 \%),{ }^{16}$ carcinoembryonic antigen $(11 \%)^{17}$ and cancer antigen (CA)-125 $(57 \%)^{18}$ at similar specificity cut-off points (94-100\%).

In both studies the sensitivity of soluble mesothelin was limited by the lack of reactivity in sarcomatoid tumours ${ }^{915}$ and some of the "mixed" mesotheliomas, presumably ones with a heavy sarcomatoid component. In both studies the sensitivity and specificity were calculated using a cutoff value determined in the same cohort. The cut-off values have not been tested in a separate cohort to confirm the sensitivity and specificity.

False positives also occur: ${ }^{5}$ soluble mesothelin is released in significant quantities by ovarian and pancreatic carcinomas, as well as by a small proportion of lung carcinomas and, in the current series, a patient with lymphoma. ${ }^{15}$ Immunohistochemistry has revealed mesothelin staining in several types of non-mesothelioma cancers $^{19}$ such as tumours of the gastrointestinal tract. It is likely that some of these tumours will shed mesothelin into the blood or serosal fluids. Because of the significantly higher incidence of malignant ascites from ovarian carcinomas, the value of soluble mesothelin in peritoneal fluid as a diagnostic marker for mesothelioma will be lower than in pleural effusions.

Pleural fluid levels of soluble mesothelin were significantly higher than-but strongly correlated to-corresponding serum levels in the study by Creaney et al. ${ }^{15}$ This would be consistent with the belief that mesothelin is released from pleural mesothelioma cells into the pleural effusion and probably absorbed subsequently into the systemic circulation. As such, pleural fluid levels of soluble mesothelin may be a more sensitive test than serum levels in diagnosing mesothelioma. Measurement of soluble mesothelin levels in both pleural fluid and serum may further increase the sensitivity.

The level of soluble mesothelin, either in the blood or pleural fluid, did not predict survival when adjusted for the histology. ${ }^{8} 15$ However, it may reflect tumour load as a rapid reduction in mesothelin levels was observed after debulking surgery for peritoneal mesothelioma. ${ }^{10} \mathrm{~A}$ biomarker that reflects the tumour response to mesothelioma treatment would be extremely 
useful, especially in research settings, as radio-logical parameters are difficult to follow in mesothelioma because of the presence of effusions or prior pleurodesis. These aspects of soluble mesothelin require confirmation in large prospective studies.

Interestingly, soluble mesothelin has made its way into clinical practice as a biomarker when the biology of mesothelin is still relatively unknown. This molecule has a role in mediating cell adhesions, and strategies to antagonise mesothelin have shown promise as innovative treatments for mesothelioma. ${ }^{12}$ Phase I trials of anti-mesothelin treatment have been completed and more advanced clinical trials are underway. Targeting mesothelin may also allow the development of novel radiological imaging techniques to "home in" specifically on the mesothelium and mesotheliomas.

\section{How would mesothelin fit into the diagnostic algorithm for exudative effusions?}

A definitive and watertight diagnosis of mesothelioma is important for determining treatment regimes (eg, pemetrexed) and securing compensation claims. Soluble mesothelin, with its limitations, cannot replace histocytological diagnosis as the "gold standard". However, it may offer additional value to the existing diagnostic tests for pleural effusions. It provides a significantly better sensitivity than cytological examination. An increased pleural fluid level of mesothelin, though not diagnostic on its own, would raise a strong suspicion of mesothelioma or metastatic (especially ovarian or pancreatic) carcinomas and would help direct further investigations (eg, thoracoscopy). Indeed, seven patients in the study by Creaney et al $^{15}$ had a raised mesothelin level in their effusions up to 10 months before the diagnosis was established. A raised soluble mesothelin level may also provide supportive evidence towards a diagnosis of mesothelioma in cases where the histopathology is inconclusive or in patients unsuitable for invasive biopsies.

Measuring the pleural fluid level of soluble mesothelin is convenient as most patients will undergo thoracentesis in the investigation of their effusions. Soluble mesothelin can be assayed in stored effusion samples, which will allow clinicians to retrospectively request the test should the initial investigation of the effusions fail to reveal a diagnosis. The commercially available enzyme-linked immunoabsorbent assay has been shown to be reliable for measurement of soluble mesothelin. ${ }^{11}$

Soluble mesothelin is a useful marker for epithelioid mesothelioma but not for other histological variants of mesothelioma. The charge now is to seek an equivalent marker for sarcomatoid mesothelioma to be used in combination with soluble mesothelin, which will vastly enhance its clinical usefulness. Attempts to improve the diagnostic yield by using better antibodies are also underway. ${ }^{12}{ }^{13}$

An ideal diagnostic marker with perfect accuracy will never be found. Soluble mesothelin represents a significant step towards the goal of diagnosing the cause of pleural effusions without involving invasive procedures.

Thorax 2007;62:561-562.

doi: $10.1136 /$ thx. 2006.076612

Correspondence to: Dr Y C Gary Lee, Oxford Centre for Respiratory Medicine, Churchill Hospital, Oxford OX3 7ப, UK; ycgarylee@ hotmail.com

Dr Lee is supported by a Wellcome Advanced Fellowship and by project grants from the Medical Research Council (UK), British Lung Foundation and NH\&MRC (Australia).

\section{REFERENCES}

1 Light RW, Lee YCG. Textbook of pleural diseases. London: Arnold Press, 2003.
2 Lee YCG, Davies RJO, Light RW. Diagnosing pleural effusion: moving beyond transudateexudate separation. Chest 2007;131:942-3.

3 Perez-Rodriguez E, Jimenez Castro D. The use of adenosine deaminase and adenosine deaminase isoenzymes in the diagnosis of tuberculous pleuritis. Curr Opin Pulm Med 2000;6:259-66.

4 West SD, Lee YCG. Current management of malignant pleural mesothelioma. Clin Chest Med 2006;27:335-54.

5 Scherpereel A, Lee YCG. Biomarkers for mesothelioma. Curr Opin Pulm Med 2007;13 (in press).

6 Kettunen E, Nicholson AG, Nagy B, et al. LICAM, INP10, P-cadherin, IPA and ITGB4 over-expression in malignant pleural mesotheliomas revealed by combined use of cDNA and tissue microarray. Carcinogenesis 2005;26:17-25.

7 Gordon GJ. Transcriptional profiling of mesothelioma using microarrays. Lung Cancer 2005;49(Suppl 1):S99-103.

8 Robinson BWS, Creaney J, Lake R, et al. Mesothelin-family proteins and diagnosis of mesothelioma. Lancet 2003;362:1612-6.

9 Scherpereel A, Grigoriu B, Conti M, et al. Soluble mesothelin-related peptides in the diagnosis of malignant pleural mesothelioma. Am J Respir Crit Care Med 2006; 173:1 155-60.

10 Hassan R, Remaley AT, Sampson ML, et al. Detection and quantitation of serum mesothelin, a tumor marker for patients with mesothelioma and ovarian cancer. Clin Cancer Res 2006; 12:447-53.

11 Beyer HL, Geschwindt RD, Glover CL, et al. MESOMARK: a potential test for malignant pleural mesothelioma. Clin Chem 2007;53:666-72.

12 Hassan R, Bera T, Pastan I. Mesothelin: a new target for immunotherapy. Clin Cancer Res 2004; 10:3937-42

13 Hellstrom I, Raycraft J, Kanan S, et al. Mesothelin variant 1 is released from tumor cells as a diagnostic marker. Cancer Epidemiol Biomarkers Prev 2006;15:1014-20.

14 Ho $M$, Onda M, Wang QC, et al. Mesothelin is shed from tumor cells. Cancer Epidemiol Biomarkers Prev 2006; 15:1751.

15 Creaney J, Yeoman D, Naumoff LK, et al. Soluble mesothelin in effusions: a useful tool for the diagnosis of malignant mesothelioma. Thorax 2007;62:569-76.

16 Thompson IM, Ankerst DP, Chi C, et al. Operating characteristics of prostate-specific antigen in men with an initial PSA level of $3.0 \mathrm{ng} / \mathrm{ml}$ or lower. JAMA 2005;294:66-70.

17 Goldberg EM, Simunovic LM, Drake SL, et al. Comparison of serum CA 19-9 and CEA levels in a population at high risk for colorectal cancer. Hybridoma 1989;8:569-75.

18 Helzlsover KJ, Bush TL, Alberg AJ, et al. Prospective study of serum CA-125 levels as markers of ovarian cancer. JAMA 1993;269:1123-6.

19 Ordonez NG. Value of mesothelin immunostaining in the diagnosis of mesothelioma. Mod Pathol 2003;16:192-7. 
Pulmonary coagulopathy

\title{
Pulmonary coagulopathy: a potential therapeutic target in different forms of lung injury
}

\author{
Marcus J Schultz, Marcel Levi
}

The role and source of tissue factor

A large body of evidence has shown that systemic coagulopathy is a key event in severe systemic inflammation, such as that which occurs in sepsis. ${ }^{1}$ Systemic coagulopathy is the net result of activation of coagulation and defective systems of natural inhibition of coagulation, on the one hand, and attenuation of fibrinolysis on the other. Activation of coagulation is primarily driven by the extrinsic coagulation pathway which starts with expression of tissue factor (TF) on mononuclear cells and endothelial cells. TF then binds and activates factor VII which activates downstream coagulation cascades. ${ }^{23}$ Mechanisms that regulate the coagulation pathway under normal conditions involve natural inhibitors of coagulation, including activated protein C (APC), antithrombin (AT) and tissue factor pathway inhibitor (TFPI). In general, they all interfere with the TFfactor VIIa-induced activation of coagulation, but on different levels. In patients with sepsis, increased coagulant activity is not sufficiently counterbalanced by these natural inhibitors. In addition, a rapid sustained increase in synthesis of plasminogen activator inhibitor (PAI)- 1 is present during the septic response. PAI- 1 is the main inhibitor of tissue-type and urokinase-type plasminogen activator (tPA and UPA) which activate the fibrinolytic system. The importance of systemic coagulopathy with sepsis has been established in experimental studies and in the randomised, prospective, doubleblind, placebo-controlled PROWESS trial in which infusion of recombinant human (rh)-APC resulted in improved survival of patients with severe sepsis. ${ }^{4}$

\section{LOCAL COAGULOPATHY WITH ACUTE LUNG INJURY}

Coagulopathy with acute lung injury and/or pneumonia

Recent studies have clearly shown that prominent changes in local fibrin turnover are an important feature of acute lung injury (ALI)/acute respiratory distress syndrome (ARDS) and pulmonary infec- tion. The profile and extent of these changes vary with the severity of inflammation: in severe pneumonia demanding mechanical ventilation the changes are nearly identical to those in ARDS, ${ }^{5}$ while less prominent alterations of alveolar fibrin turnover have been measured in spontaneously breathing patients with pneumonia. ${ }^{5}$ The mechanisms that contribute to disturbed alveolar fibrin turnover are not clearly understood, but are thought to be similar to those found in the intravascular spaces during severe systemic inflammation. ${ }^{7-9}$ Similar to sepsis, in ARDS and pneumonia alveolar thrombin generation seems to be mediated by the TF-factor VIIa pathway. Patients who develop ventilator-associated pneumonia have increased bronchoalveolar levels of soluble TF and factor VII. ${ }^{9}$ In patients with ARDS an increase in soluble TF, factor VIIa and TF-dependent factor $\mathrm{X}$ activation in bronchoalveolar lavage (BAL) fluid has been demonstrated. In addition, inhibition of the TF-factor VIIa pathway completely abrogated intrapulmonary fibrin deposition in patients with ARDS. ${ }^{10}$ Although the lung has only a limited capacity to produce protein C, APC is present in BAL fluid. ${ }^{11}$ The protein $C$ system has been shown to be suppressed in patients with ventilatorassociated pneumonia ${ }^{12}{ }^{13}$ and pulmonary inflammation. $^{14}$ In association with enhanced fibrin production, fibrinolytic activity is depressed in BAL fluid of patients with ALI/ARDS or pneumonia, related to high pulmonary concentrations of PAI-1. PAI-1 is increased in ALI/ARDS and is probably secreted by lung epithelial cells, fibroblasts and endothelial cells..$^{15} 16$ Patients at risk of ventilator-associated pneumonia show similar changes in pulmonary fibrin turnover. ${ }^{9}$ The important role of the fibrinolytic system in the pathogenesis of pneumonia is underscored by the observation that the depression of bronchoalveolar fibrinolysis precedes the clinical occurrence of ventilator-associated pneumonia by several days. ${ }^{9}$

\section{Ventilator-induced coagulopathy}

Compelling evidence from preclinical and clinical studies shows that mechanical ventilation aggravates or may even initiate lung injury. ${ }^{17}{ }^{18}$ The similarities between the inflammatory responses in pneumonia and ALI/ARDS suggest that similar changes in coagulation and fibrinolysis may occur in ventilator-induced lung injury. The knowledge on ventilator-induced coagulopathy is rapidly growing. Several preclinical studies and one clinical study in healthy subjects suggest that pulmonary fibrin turnover is indeed influenced by mechanical ventilation. ${ }^{19-21}$ Some suggestion on the existence of TF-mediated coagulation caused by mechanical ventilation comes from a preliminary report on short-term mechanical ventilation in patients undergoing a surgical procedure. $^{21}$ In addition, small but consistent changes in pulmonary coagulation in mice have been found with injurious forms of mechanical ventilation (Wolthuis, unpublished data). Only two preclinical studies have focused on the effect of mechanical ventilation on pulmonary fibrinolysis, ${ }^{19} 20$ in which it was found that injurious mechanical ventilation attenuates fibrinolytic activity in rats, which appears to be caused by increased production and/ or release of PAI- 1 .

\section{SOURCE OF TF IN ALVEOLAR SPACES}

One limitation with all studies on pulmonary coagulopathy so far is that they do not give sufficient insight into the potential sources of TF in the alveolar spaces. It has been suggested that changes in coagulation and fibrinolysis found in the pulmonary compartment are the result of leakage of coagulation factors into the lungs from the systemic circulation. Indeed, concordant increased activation of coagulation in the systemic compartment was found with infectious pulmonary coagulopathy in one study. ${ }^{9}$ However, TF is not present in the systemic circulation. In addition, pulmonary infection was characterised by local (but not systemic) attenuation of fibrinolysis, suggesting that pulmonary coagulopathy is a localised process.

In this issue of Thorax, Bastarache et al ${ }^{22}$ report their findings on the ability of the alveolar epithelium to initiate intra-alveolar coagulation by expressing active TF (see page 608). Using an in vitro cell surface TF assay and TF ELISA, they measured the production and activity of TF in cultured alveolar epithelial cells following exposure to different stimuli. TF activity, mRNA and protein levels increased in A549 cells after stimulation with a pro-inflammatory stimulus (cytomix). Importantly, increased TF activity was also measured following incubation with pulmonary oedema fluid from 
patients with ALI/ARDS. Furthermore, immunohistochemistry for TF in human lung tissue from patients with ALI/ARDS showed prominent TF staining in alveolar epithelial cells as well as intra-alveolar macrophages and hyaline membranes. Given the markedly increased levels in pulmonary oedema fluid compared with plasma, it strongly suggests that there is an intra-alveolar source of TF. The reported findings further provide convincing evidence that the alveolar epithelium can initiate TF-dependent intra-alveolar coagulation in this disease setting. The major strengths of the study include the use of a well-characterised and large cohort of patients with ALI/ARDS with an appropriate control group, the use of co-staining with a type II epithelial cell marker in the immunohistochemical studies and the fact that TF measurements were performed on undiluted pulmonary oedema fluid rather than using BAL fluid, thus avoiding potential complications due to dilution effects. One weakness of the study is that the investigators did not study alveolar macrophages, as it has been shown that alveolar macrophages from humans contain procoagulant activity. ${ }^{23}$ Also, they did not consider the pulmonary vascular endothelium as a potential source of TF during pulmonary inflammation.

\section{CLINICAL CONSIDERATIONS}

Infusion of rh-APC has been found to reduce mortality of patients with severe sepsis. ${ }^{4}$ Many patients included in this trial had concomitant ARDS, and many had pneumonia as the primary source of sepsis. ${ }^{24}$ One can hypothesise that the beneficial effect of APC was the result, at least in part, of APC on intra-alveolar coagulopathy. Indeed, rh-APC exerts anticoagulant effects in the human lung challenged with endotoxin: ${ }^{25}$ activation of coagulation after pulmonary challenge with endotoxin is inhibited and increased PAI- 1 activity is diminished by infusion of rh-APC. Thus, systemic administration of APC influences pulmonary fibrin turnover.

Clinical trials in ALI/ARDS have primarily targeted inflammation and not coagulation, although nebulisation of heparin has been found to be beneficial in preclinical and clinical studies in patients with inhalation trauma. ${ }^{26-30}$ The results from new studies on interventions on (alveolar) fibrin turnover with TFPI and rh-APC are presently awaited. In view of the results from the study by Bastarache et $a l^{22}$ epithelial-directed treatment that could be delivered by inhalation of nebulised anticoagulant or profibrinolytic agents seems an attractive approach in treating patients with or at risk of pulmonary coagulopathy.

\section{CONCLUSION}

Alveolar fibrin deposition is an important feature of pulmonary infection or inflammation. Some studies also suggest that pulmonary coagulopathy is a feature of ventilator-induced lung injury. Mechanisms that contribute to this fibrin deposition are localised thrombin generation and depression of bronchoalveolar fibrinolysis. Remarkably, changes in alveolar coagulation and fibrinolysis closely resemble those found systemically in patients with sepsis. Recent studies have demonstrated the beneficial effect of anticoagulant therapy in sepsis. Theoretical considerations suggest novel therapeutic strategies or preventive measures in critically ill patients, but clinical studies are needed to examine this hypothesis.

Thorax 2007;62:563-564.

doi: $10.1136 /$ thx.2006.067256

\section{Authors' affiliations}

Marcus J Schultz, Department of Intensive Care Medicine and Laboratory of Experimental Intensive Care and Anesthesiology (LEICA), Academic Medical Center - University of Amsterdam, and HERMES Critical Care Group, Amsterdam, The Netherlands

Marcel Levi, Department of Internal Medicine, Academic Medical Center - University of Amsterdam, Amsterdam, The Netherlands

Correspondence to: Dr Marcus J Schultz, Department of Intensive Care Medicine, C3-415, Academic Medical Center, Meibergdreef 9, 1105 AZ Amsterdam, The Netherlands; m.j.schultz@amc.uva.nl

Competing interests: None.

\section{REFERENCES}

1 Levi M, Ten Cate H. Disseminated intravascular coagulation. N Engl J Med 1999;341:586-92.

2 Levi M, ten Cate H, Baver KA, et al. Inhibition of endotoxin-induced activation of coagulation and fibrinolysis by pentoxifylline or by a monoclonal anti-tissue factor antibody in chimpanzees. J Clin Invest 1994;93:114-20.

3 Giesen PL, Rauch U, Bohrmann B, et al. Bloodborne tissue factor: another view of thrombosis. Proc Natl Acad Sci USA 1999;96:231 1-5.

4 Bernard GR, Vincent JL, Laterre PF, et al. Efficacy and safety of recombinant human activated protein C for severe sepsis. N Engl J Med 2001;344:699-709.

5 Gunther A, Mosavi P, Heinemann S, et al. Alveolar fibrin formation caused by enhanced procoagulant and depressed fibrinolytic capacities in severe pneumonia. Comparison with the acute respiratory distress syndrome. Am J Respir Crit Care Med 2000; 161:454-62.

6 Fuchs-Buder T, de Moerloose P, Ricou B, et al. Time course of procoagulant activity and D dimer in bronchoalveolar fluid of patients at risk for or with acute respiratory distress syndrome. Am J Respir Crit Care Med 1996;153:163-7.

7 Idell S, Gonzalez K, Bradford H, et al. Procoagulant activity in bronchoalveolar lavage in the adult respiratory distress syndrome. Contribution of tissue factor associated with factor VII. Am Rev Respir Dis 1987; 136:1466-74

8 Idell S, Koenig KB, Fair DS, et al. Serial abnormalities of fibrin turnover in evolving adult respiratory distress syndrome. Am J Physiol 1991;261:L240-8.

9 Schultz MJ, Millo J, Levi M, et al. Local activation of coagulation and inhibition of fibrinolysis in the lung during ventilator associated pneumonia. Thorax 2004;59:130-5.

10 Miller DL, Welty-Wolf K, Carraway MS, et al. Extrinsic coagulation blockade attenuates lung injury and proinflammatory cytokine release after intratracheal lipopolysaccharide. Am J Respir Cell Mol Biol 2002;26:650-8.

11 Yamamoto K, Loskutoff DJ. Extrahepatic expression and regulation of protein $C$ in the mouse. Am J Pathol 1998; 153:547-55.

12 Choi G, Schultz MJ, Levi M, et al. Protein C in pneumonia. Thorax 2005:60:705-6.

13 Choi G, Bresser P, Levi M, et al. Disturbed alveolar fibrin turnover during pneumonia is associated with reduced activated protein $\mathrm{C}$ levels in lungs. Crit Care 2004;8(Suppl 1):111.

14 Hataji O, Taguchi O, Gabazza EC, et al. Activation of protein $C$ pathway in the airways. Lung 2002; 180:47-59.

15 Idell S. Endothelium and disordered fibrin turnover in the injured lung: newly recognized pathways. Crit Care Med 2002;30:S274-80.

16 Marshall BC, Sageser DS, Rao NV, et al. Alveolar epithelial cell plasminogen activator. Characterization and regulation. J Biol Chem 1990;265:8198-204.

17 Belperio JA, Keane MP, Burdick MD, et al. Critical role for CXCR2 and CXCR2 ligands during the pathogenesis of ventilator-induced lung injury. J Clin Invest 2002;110:1703-16.

18 Altemeier WA, Matute-Bello G, Frevert CW, et al. Mechanical ventilation with moderate tidal volumes synergistically increases lung cytokine response to systemic endotoxin. Am J Physiol Lung Cell Mol Physiol 2004;287:L533-42.

19 Dahlem P, Bos AP, Haitsma JJ, et al. Alveolar fibrinolytic capacity suppressed by injurious mechanical ventilation. Intensive Care Med 2005;31:724-32.

20 Dahlem P, Bos AO, Haitsma JJ, et al. Mechanical ventilation affects alveolar fibrinolysis in LPSinduced lung injury. Eur Respir J 2006;28:992-8.

21 Choi G, Wolthuis E, Bresser P, et al. Mechanical ventilation with lower tidal volumes and positive end-expiratory pressure prevents alveolar coagulation in patients without lung injury. Anesthesiology 2006;105:689-95.

22 Bastarache J, Wang L, Geiser T, et al. The alveolar epithelium can initiate the extrinsic coagulation cascade through expression of tissue factor. Thorax 2007;62:608-16.

23 Lyberg T, Nakstad B, Hetland O, et al. Procoagulant (thromboplastin) activity in human bronchoalveolar lavage fluids is derived from alveolar macrophages. Eur Respir J 1990;3:61-7.

24 Laterre P-F, Garber G, Levy H, et al. Severe community-acquired pneumonia as a cause of severe sepsis: data from the PROWESS study. Crit Care Med 2005;33:952-61

25 van der Poll T, Levi M, Nick JA, et al. Activated protein $\mathrm{C}$ inhibits local coagulation after intrapulmonary delivery of endotoxin in humans. Am J Respir Crit Care Med 2005;171:1 125-8.

26 Desai MH, Mlcak R, Richardson J, et al. Reduction in mortality in pediatric patients with inhalation injury with aerosolized heparin/N-acetylcystine [correction of acetylcystine] therapy. J Burn Care Rehabil 1998;19:210-2.

27 Brown M, Desai M, Traber LD, et al. Dimethylsulfoxide with heparin in the treatment of smoke inhalation injury. J Burn Care Rehabil 1988;9:22-5.

28 Cox CS Jr, Zwischenberger JB, Traber DL, et al. Heparin improves oxygenation and minimizes barotrauma after severe smoke inhalation in an ovine model. Surg Gynecol Obstet 1993;176:339-49.

29 Murakami K, McGuire R, Cox RA, et al. Heparin nebulization attenuates acute lung injury in sepsis following smoke inhalation in sheep. Shock 2002;18:236-41.

30 Saliba MJ Jr. The effects and uses of heparin in the care of burns that improves treatment and enhances the quality of life. Acta Chir Plast 1997;39:13-6. 